\title{
A!
}

This is an electronic reprint of the original article.

This reprint may differ from the original in pagination and typographic detail.

Kamppinen, T.; Mäkinen, J. T.; Eltsov, V. B.

\section{Dimensional control of tunneling two-level systems in nanoelectromechanical resonators}

Published in:

Physical Review B

DOI:

10.1103/PhysRevB.105.035409

Published: 15/01/2022

Document Version

Publisher's PDF, also known as Version of record

Please cite the original version:

Kamppinen, T., Mäkinen, J. T., \& Eltsov, V. B. (2022). Dimensional control of tunneling two-level systems in nanoelectromechanical resonators. Physical Review B, 105(3), 1-11. [035409].

https://doi.org/10.1103/PhysRevB.105.035409

This material is protected by copyright and other intellectual property rights, and duplication or sale of all or part of any of the repository collections is not permitted, except that material may be duplicated by you for your research use or educational purposes in electronic or print form. You must obtain permission for any other use. Electronic or print copies may not be offered, whether for sale or otherwise to anyone who is not an authorised user. 


\title{
Dimensional control of tunneling two-level systems in nanoelectromechanical resonators
}

\author{
T. Kamppinen $\odot,{ }^{*}$ J. T. Mäkinen $\odot$, and V. B. Eltsov $\odot$ \\ Low Temperature Laboratory, Department of Applied Physics, Aalto University, FI-00076 Aalto, Finland
}

(Received 25 October 2021; revised 21 December 2021; accepted 21 December 2021; published 7 January 2022)

Tunneling two-level systems affect damping, noise, and decoherence in a wide range of devices, including nanoelectromechanical resonators, optomechanical systems, and qubits. Theoretically, this interaction is usually described within the tunneling state model. The dimensions of such devices are often small compared to the relevant phonon wavelengths at low temperatures, and extensions of the theoretical description to reduced dimensions have been proposed, but lack conclusive experimental verification. We have measured the intrinsic damping and the frequency shift in magnetomotively driven aluminum nanoelectromechanical resonators of various sizes at millikelvin temperatures. We find good agreement of the experimental results with a model where the tunneling two-level systems couple to flexural phonons that are restricted to one or two dimensions by geometry of the device. This model can thus be used as an aid when optimizing the geometrical parameters of devices affected by tunneling two-level systems.

DOI: 10.1103/PhysRevB.105.035409

\section{INTRODUCTION}

Modern nanofabrication techniques allow unique and extremely sensitive mechanical probes of force and mass with widespread applications as sensors, actuators, parametric amplifiers, and in fundamental physics. For example, magnetic force microscopy with single spin resolution [1] and the quantum ground state of motion [2] have already been realized. Micro- and nanoelectromechanical systems (MEMS and NEMS, respectively) are also emerging in studies of the superfluids ${ }^{3} \mathrm{He}$ and ${ }^{4} \mathrm{He}$ [3-9], where they hold promise for superior sensitivity and spatial resolution over the immersed quartz tuning forks [10] and vibrating wires [11] routinely used in cryogenic research. Measuring the dynamics of a single quantized vortex in the superfluids is feasible with NEMS resonators [12,13]. Detailed analysis of such high-precision measurements requires a thorough understanding of the intrinsic damping mechanisms of the devices.

NEMS devices benefit from the low temperatures used in cryogenic experiments through the decrease in thermal noise and in intrinsic damping mechanisms [14]. However, as the size of the devices goes down, the $Q$ value tends to decrease [15], which is generally attributed to the increase in the surface-area-to-volume ratio. At low temperatures, the observed damping and frequency shift of many NEMS devices have been explained in the framework of the tunneling state model (TSM), which was originally developed to describe properties of bulk amorphous materials at low temperatures $[16,17]$. For sufficiently small devices, and as the temperature is reduced, the thermal phonon wavelength becomes larger than the characteristic dimensions of the device and the bulk model of the TSM theory is no longer valid. In such quasi-one-dimensional (1D) and quasi-two-dimensional

*timo.kamppinen@aalto.fi
(2D) geometries, restrictions on the allowed phonon modes need to be accounted for [18]. With development in quantum technologies, the size of the devices continues to decrease, and thus research on tunneling two-level systems (TTLSs) in reduced dimensions has emerged as its own field. While many experimental works address TTLSs in NEMS and MEMS resonators with reduced dimensions, Refs. [19-32], the experimental demonstration and matching with the theory, both in $1 \mathrm{D}$ and in $2 \mathrm{D}$ cases, in the devices of the same type have so far been missing.

In this paper, we present our experimental results on aluminum NEMS resonators hosting TTLSs that couple to dispersive phonon modes, restricted by geometry to $1 \mathrm{D}$ and 2D. Typical devices can be seen in Fig. 1. The experiments have been performed in vacuum at temperatures between $16 \mathrm{mK}$ and $4 \mathrm{~K}$. In this temperature range, the devices exhibit three damping mechanisms: TTLSs, magnetomotive, and clamping damping. Of these, the magnetomotive and clamping mechanisms are temperature independent, while the TTLS damping is expected to scale as $\Gamma_{1 \mathrm{D}} \propto T^{1 / 2}$ or $\Gamma_{2 \mathrm{D}} \propto T$ [18]. The observed temperature dependence of the response of the devices is in good agreement with theoretical expectations.

The paper is structured as follows: We first explain the fabrication and measurement scheme of the nanomechanical devices, we then introduce the dynamics equation relevant to the experiments and the theory regarding magnetomotive loading and TTLSs. The theory is followed by the experimental results and the conclusions.

\section{METHODS}

\section{A. Fabrication process}

Four aluminum NEMS resonators N1, N2, W1, and W2 have been studied in this paper. N1 and N2 are narrow devices while W1 and W2 are wide devices, see Fig. 1. The П-shaped 

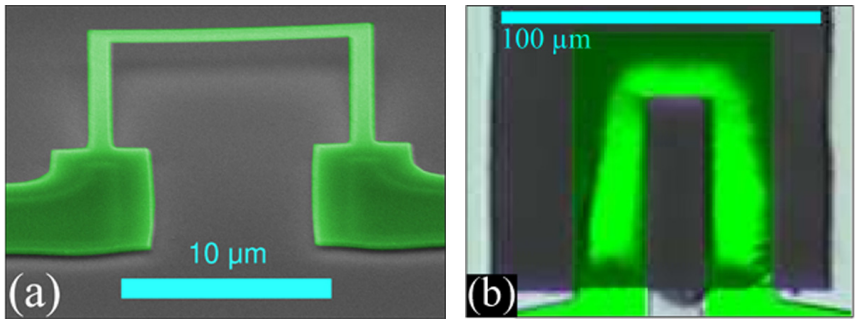

FIG. 1. (a) False-color scanning electron micrograph of the device N1 (green). The view is tilted from the normal incidence by $70^{\circ}$, so the normal of the silicon surface (dark grey) points up and out of the page. The vibrating element is aluminum and comprises the two cantilever beams (legs) connected by a third beam (paddle), suspended above the silicon substrate. The wider aluminum sections are anchored on top of a silicon oxide layer and are used to carry the electrical signals required for measuring the motion of the device. The visible bending of the device away from the silicon surface results from the built-in stresses that have relieved when the device is released. (b) False-color optical micrograph of the device W2 (green), viewed from the normal incidence on the silicon surface. The vibrating element is suspended above an orifice (black) in the silicon substrate (gray) while the rest of the structure is anchored on top of the silicon oxide layer. The wrinkles, visible especially close to the clamped end of the feet, result from compressive stress in the silicon oxide window prior to the release etch.

geometry of the devices comprises two rectangular cantilever feet connected by a rectangular paddle, as shown in Fig. 2. The dimensions of the devices are shown in Table I. The devices are designed to be used as sensors immersed in a superfluid, and the geometry was inspired by the work in Ref. [4]. The benefits of the geometry are low spring constants for detecting tiny forces associated, e.g., with quantized vortices, low frequency for low acoustic losses in fluids, and large surface area for high sensitivity to excitations in the superfluids.

The fabrication process of the aluminum NEMS devices is similar to that described in Ref. [13]. Devices N1 and N2 have been fabricated on high purity (resistivity $>100 \Omega \mathrm{m}$ ) silicon

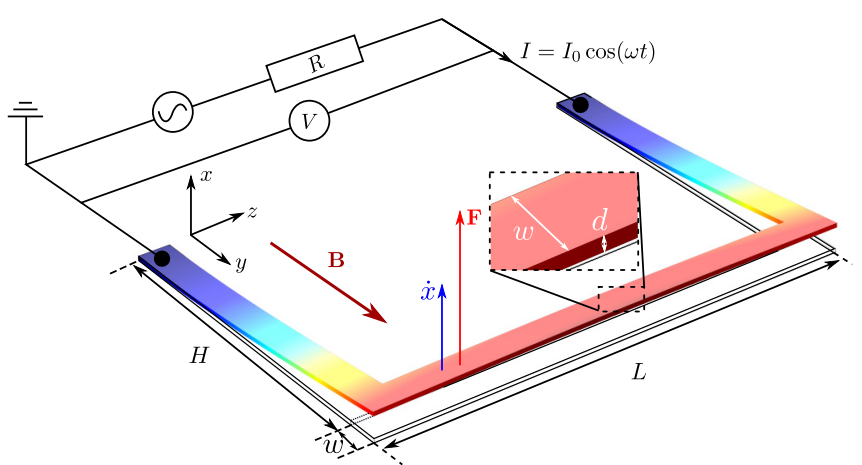

FIG. 2. The fundamental eigenmode of the resonator, composed of two cantilever feet, connected with the rigid paddle. The mode shape is obtained from Comsol simulation and the colors represent the relative displacement with respect to the stationary frame. The magnetomotive drive $(\mathbf{B}, I, \mathbf{F})$ and detection $(V, \dot{x})$ scheme, the geometrical parameters $(H, L, w, d)$ and the coordinate system $(x$, $y, z)$ used in this paper are also depicted.
TABLE I. The properties of the devices studied in this paper. The dimensions $L, H, w, d$ are shown in Fig. 2. The resonance frequency $f_{0}=\omega_{0} /(2 \pi)$ is measured at the cryostat base temperature. $P_{s}=10^{-44} P_{0}$ is the scaled TTLS density of states (with the units $\mathrm{J}^{-1} \mathrm{~m}^{-3}$ ), which together with the TTLS-phonon coupling parameter $\gamma$ are obtained from fits to the measured damping. The product $P_{0} \gamma^{2}$ is obtained separately from fits to the damping and to the frequency shift (units are $\mathrm{MJ} / \mathrm{m}^{3}$ ). The parameters $a_{m}$ and $\Delta f_{c}$ describe contributions to the damping from the magnetomotive effect and clamping, respectively.

\begin{tabular}{lcccc}
\hline \hline Device & $\mathrm{N} 1$ & $\mathrm{~N} 2$ & $\mathrm{~W} 1$ & $\mathrm{~W} 2$ \\
\hline$L(\mu \mathrm{m})$ & 14.7 & 28.9 & 60 & 60 \\
$H(\mu \mathrm{m})$ & 13.0 & 13.2 & 44 & 60 \\
$w(\mu \mathrm{m})$ & 1.11 & 1.10 & 20 & 20 \\
$d(\mathrm{~nm})$ & $150 \pm 8$ & $150 \pm 8$ & $200 \pm 10$ & $200 \pm 10$ \\
$f_{0}(\mathrm{kHz})$ & 395.2 & 291.8 & 73.8 & 68.0 \\
$P_{s}$ & $0.49 \pm 0.05$ & $0.60 \pm 0.06$ & $7.5 \pm 1.3$ & $6.3 \pm 1.5$ \\
$\gamma(\mathrm{eV})$ & $2.9 \pm 0.1$ & $2.6 \pm 0.1$ & $0.93 \pm 0.04$ & $0.78 \pm 0.05$ \\
$\left.P_{0} \gamma^{2}\right|_{\text {damping }}$ & $10.6 \pm 0.2$ & $10.2 \pm 0.6$ & $17 \pm 2$ & $10 \pm 2$ \\
$\left.P_{0} \gamma^{2}\right|_{\text {frequencyshift }}$ & $4.7 \pm 0.4$ & $4.8 \pm 0.5$ & $11.1 \pm 0.2$ & $4.9 \pm 0.3$ \\
$a_{m}\left(\mathrm{~Hz} / \mathrm{T}^{2}\right)$ & $630 \pm 180$ & $460 \pm 260$ & $710 \pm 14$ & $387 \pm 13$ \\
$\Delta f_{c}(\mathrm{~Hz})$ & - & - & $12 \pm 3$ & $4 \pm 3$ \\
\hline \hline
\end{tabular}

substrates with a 275 -nm-thick sacrificial silicon oxide layer on top. Devices W1 and W2 have been fabricated on commercially available substrates $[33,34]$ where the sacrificial silicon oxide layer is $40 \mathrm{~nm}$ thick and is suspended above a rectangular orifice in the silicon substrate. The devices have been patterned by means of electron beam lithography on a positive tone PMMA bilayer resist stack. The aluminum layer has been deposited with an electron beam evaporator at pressures below $10^{-7} \mathrm{mbar}$. To release the aluminum resonator, an isotropic dry HF-vapor etch process (Primaxx MEMS-CET) has been used.

The stress acquired in the structures during the metal deposition is mostly relieved when the devices are released. Consequently, the devices typically bend away from the silicon surface, as can be seen in Fig. 1(a). When a device is cooled down, the aluminum beams contract more than the silicon substrate, and bending stress is formed in the legs that are pulled toward each other by the contracting paddle. The stress is expected to increase the resonance frequency, and measurements show that the resonance frequency is typically 5-10 \% higher at $4 \mathrm{~K}$ temperature than at room temperature. Compared to the results obtained for aluminum doubly clamped beams in Ref. [21], where the calculated increase in frequency resulting from the strain is $30-70 \%$, the observed frequency shift in the $\Pi$-shaped devices is relatively small. We note that, in general, stress in the structures changes the phonon dispersion relations via acoustoelastic effects [35], which should be taken into account when making predictions from the TTLS theory. The $\Pi$-shaped geometry of the devices allows us to test the validity of the TTLS models [18] in a configuration where stress-induced changes in the dispersion relations are relatively small. In the experimental temperature range considered here, the thermal expansion coefficients are small, and between $4 \mathrm{~K}$ and $16 \mathrm{mK}$ the calculated relative 
increase in frequency is about $10^{-7}$, which is negligible compared to other effects in this temperature range.

The silicon oxide windows, on which devices $\mathrm{W} 1$ and W2 were fabricated, were under compressive stress and had wrinkles. These wrinkles remained in the final devices after the release etch, and are also visible in the optical micrograph in Fig. 1(b). We haven't analyzed how the wrinkles affect the mechanical response of the devices but they could, in principle, have a negative impact on the mechanical $Q$ values and they might also contribute to the higher-than-expected resonance frequencies. We expect that the effect of the wrinkles is independent of the temperature in the experimental temperature range below $4 \mathrm{~K}$.

\section{B. Measurement scheme}

The motion of the NEMS resonator is actuated and measured electrically in an applied static magnetic field. The magnetomotive measurement scheme is depicted in Fig. 2. When the NEMS device, lying in the $y z$ plane, is placed in a constant magnetic field $\mathbf{B}=B \hat{\mathbf{y}}$ and an ac excitation current $I=I_{0} \cos (\omega t)$ is fed through the device, the paddle experiences a Lorentz force:

$$
\mathbf{F}(t)=I_{0} L B \cos (\omega t) \hat{\mathbf{x}} .
$$

The motion of the paddle through the magnetic field induces an electromotive force (voltage),

$$
U(t)=L B \dot{x}(t),
$$

between the two ends of the beam. The devices are operated at the fundamental eigenmode, where the two cantilever feet move out of plane symmetrically, and the paddle between them is practically rigid (i.e., the velocity profile over the length of the paddle is uniform), allowing the use of the simple expression, Eq. (2), for the generated voltage.

To measure the response of a device, we use a four-wire measurement, where one pair of wires carries the excitation current and another pair is used to measure the voltage over the device. The sinusoidal excitation current $I$ is produced by an arbitrary wave-form generator, followed by a attenuator $(40 \mathrm{~dB}$ or $80 \mathrm{~dB})$ and a resistor $R \sim 1 \mathrm{k} \Omega$ connected in series with the NEMS device. The electromotive force (voltage) generated by the motion of the device through the magnetic field is amplified with a room-temperature preamplifier and measured with a lock-in amplifier. A superconducting coil (1650 turns, inner diameter $18 \mathrm{~mm}$, height $15 \mathrm{~mm}$ ) provides a magnetic field $(83.5 \mathrm{mT} / \mathrm{A}$ at the center of the magnet) enabling the magnetomotive measurement scheme. The finite normal-state resistance of the aluminum structure, the inductance of the wires, and the capacitive coupling between the leads contribute to a background signal that is independent of the motion of the NEMS resonator. For narrow frequency sweeps around the resonance, the background signal is essentially a linear function of the frequency, and its contribution is subtracted from the measured response.

The NEMS devices are mounted in a hermetically sealed copper container, which is secured to the mixing chamber stage of a dilution refrigerator. The temperature is measured with ruthenium oxide thermometers, installed at the respective mixing chambers (the narrow and wide devices were measured in different dilution refrigerators). To verify the calibration of the thermometers, their readings were compared to the observed superfluid transition in ${ }^{4} \mathrm{He}$ (devices $\mathrm{W} 1$ and $\mathrm{W} 2$ ) and to the temperature reading obtained from a noise thermometer (devices $\mathrm{N} 1$ and $\mathrm{N} 2$ ). The ruthenium oxide thermometers used in the measurements are expected to be accurate to within $10 \%$ of the indicated temperature reading.

Since the NEMS devices reside on an insulating silicon chip, the thermalization occurs mostly via the wiring. The aluminum bond wires and the on-chip wiring are in the normal state due to the applied magnetic field. Owing to their large aspect ratio, the biggest bottleneck for the thermalization is the thin on-chip wiring. Self-heating of the NEMS devices is the most severe at the lowest temperatures where the heat conductivity is at its lowest. The main sources of heating are Joule heating and the internal friction of the devices. An estimate of the device temperature increase can be obtained by considering the typical resistance of the device with the aluminum in the normal state, which is $R=0.5 \Omega$ at low temperatures $(<5 \mathrm{~K})$. The thermal resistance of the device is obtained from the Wiedemann-Franz law $R_{T}=R /\left(L_{0} T\right)$, where $L_{0}$ is the Lorenz number, giving $R_{T} \approx 2 \times 10^{9} \mathrm{KW}^{-1}$ at $10 \mathrm{mK}$. With a typical excitation current of $1 \mu \mathrm{A}$, the Joule heating is $0.5 \mathrm{pW}$, and the expected temperature increase of the devices is $1 \mathrm{mK}$ at the lowest temperatures. The heat released due to the damping of the mechanical motion is less than $0.1 \mathrm{pW}$ with the typical oscillation amplitudes $(<100 \mathrm{~nm})$, and its contribution to the heating of the devices operated with the aluminum in the normal state is small.

\section{THEORY}

\section{A. Resonator equation of motion}

A NEMS device can be treated as a damped oscillator driven by the Lorentz force $F(t)=F_{0} \cos (\omega t)$, where $F_{0}=$ $I_{0} L B$. For most of the temperature range, the device response is linear, and the harmonic approximation is valid. At the lowest temperatures, the damping becomes small and nonlinear effects become important even at oscillation amplitudes comparable to the noise in the measurements.

Collin et al. [36] suggest that the nonlinearity of the response is of geometric origin, assuming the resonator material is in the elastic limit and the detection method is linear. In our measurements, nonlinear damping (seen as increased linewidth with increasing amplitude) is not observed at the experimental oscillation amplitudes, and the relevant dynamics equation is [36]

$$
\begin{aligned}
& m_{0}\left(1+m_{1} x+m_{2} x^{2}\right) \ddot{x}+m_{0}\left(m_{1} / 2+m_{2} x\right) \dot{x}^{2}+m_{0} \Gamma \dot{x} \\
& \quad+k\left(1+k_{1} x+k_{2} x^{2}\right) x=F_{0} \cos (\omega t),
\end{aligned}
$$

where $m_{0}$ and $k$ are the (linear) effective mass and spring constant, $\Gamma=2 \pi \Delta f$ is the damping coefficient (in units rads ${ }^{-1}$ ), $m_{i}$ and $k_{i}$ are the nonlinear mass and spring coefficients, and $x$ is the displacement from the equilibrium position. The linear resonance frequency is given by $\omega_{0}=\sqrt{k / m_{0}}$ and the quality factor is defined as $Q=\omega_{0} / \Gamma$. 
The steady-state solution of Eq. (3) is a sum of oscillating terms:

$$
x(t)=\sum_{n=0}^{\infty} x_{n}^{s} \sin (n \omega t)+x_{n}^{c} \cos (n \omega t) .
$$

In Ref. [36], the solution is found for the first harmonic $n=1$, also retaining the amplitude of the $n=2$ term:

$$
x_{1}^{s}(\omega)=\frac{F_{0}}{m_{0}} \frac{\Gamma \omega}{\left(\omega_{r}^{2}-\omega^{2}\right)^{2}+\Gamma^{2} \omega^{2}}
$$

and

$$
x_{1}^{c}(\omega)=\frac{F_{0}}{m_{0}} \frac{\omega_{r}^{2}-\omega^{2}}{\left(\omega_{r}^{2}-\omega^{2}\right)^{2}+\Gamma^{2} \omega^{2}},
$$

where the resonance position $\omega_{r}$ is a function of the squared displacement amplitude $x_{0}^{2}=\left(x_{1}^{s}\right)^{2}+\left(x_{1}^{c}\right)^{2}$ :

$$
\omega_{r}=\sqrt{\omega_{0}^{2}+2 \omega_{0} \beta x_{0}^{2}} \approx \omega_{0}+\beta x_{0}^{2},
$$

where $\beta\left(m_{1}, m_{2}, k_{1}, k_{2}\right)$ is the frequency-pulling parameter, which, to a first approximation, is a constant.

In practice, we measure the $n=1$ term while the other terms in the series in Eq. (4) are effectively rejected by the lock-in amplifier. The parameters $\omega_{0}, \Gamma$, and $\beta$ are obtained by fitting Eqs. (5) and (6) to the measured resonance responses. At small amplitudes, the nonlinear frequency shift is small compared to the line width, $\beta x_{0}^{2} \ll \Gamma$, and the resonance response is practically Lorentzian. As the amplitude at resonance increases, the amplitude-frequency curve becomes asymmetric, and at sufficiently high amplitudes the response becomes multivalued, showing hysteresis depending on the frequency sweep direction.

\section{B. Magnetomotive damping and frequency shift}

Due to the magnetomotive measurement scheme, part of the energy stored in the oscillatory motion of the mechanical resonator is lost. There are two contributions to the excess damping of the form $\Gamma_{m} \propto B^{2}$ : power dissipated in the external circuit [37], important especially for long and thin wires; and eddy current losses [38], which are important, e.g., for conducting plates moving in a magnetic field.

The increase in damping rate corresponding to the power dissipated in the external circuit is [37]

$$
\Gamma=\Gamma_{0}+\frac{L^{2} B^{2}}{m_{0}} \frac{\operatorname{Re}\left(Z_{\mathrm{ext}}\right)}{\left|Z_{\mathrm{ext}}\right|^{2}},
$$

where $\Gamma_{0}$ is the intrinsic damping rate and $Z_{\text {ext }}$ is the complex impedance of the measuring circuit. If the circuit has a reactive component, the observed resonance frequency $\omega_{m}$ shifts as well:

$$
\left(\frac{\omega_{m}}{\omega_{0}}\right)^{2}=1+\frac{L^{2} B^{2}}{\omega_{0} m_{0}} \frac{\operatorname{Im}\left(Z_{\mathrm{ext}}\right)}{\left|Z_{\text {ext }}\right|^{2}} .
$$

Another contribution to magnetic damping is eddy currents generated within the devices which we believe are important in our case (see Sec. IV A). Accurate modeling of eddy current losses even in simple geometries is in general a challenging task [39] and is beyond the scope of this paper. However, a simple estimate for our devices is given in the Appendix, Eq. (A5). Our model suggests that the losses are directly proportional to the squared magnetic field and the electrical conductivity of the device material. In the experimental temperature range, the electrical conductivity of aluminum is constant, and the eddy current losses are assumed to be independent of the temperature. For the purposes of this paper, the empirical model

$$
\Gamma=\Gamma_{0}+2 \pi a_{m} B^{2},
$$

where $a_{m}$ is a fitting parameter, is sufficient to describe our results.

\section{Tunneling two-level systems}

The physical properties of amorphous materials at low temperatures are explained in the framework of the TSM [16,17] which assumes that the material hosts a large ensemble of TTLSs with a wide range of energy splittings and almost constant density of states (DOS), typically of the order $P_{0} \sim$ $10^{44} \mathrm{~J}^{-1} \mathrm{~m}^{-3}$. In a mechanical resonator, the TTLSs couple to the applied strain fields, where the coupling is characterized by the material-dependent deformation potential $\gamma \sim 1 \mathrm{eV}$. The TTLSs absorb energy from the mechanical mode and redistribute it among the rest of the degrees of freedom of the system (phonons, electrons, or other TTLSs).

In aluminum devices, TTLSs are likely to exist in a few-nanometers-thick amorphous aluminum oxide layer covering the devices and possibly at the grain boundaries of the polycrystalline metal, rather than within the aluminum crystals [40]. It is an interesting question whether the TTLSs act as in insulating glasses (where they couple mostly to phonons) due to the insulating oxide layer, or as in metallic glasses with strong coupling to electrons due to the presence of the underlying aluminum structure, or whether signatures of both can be seen.

The reduced dimensions have important consequences to the relaxation of TTLSs in NEMS devices as the thermal phonon wavelength $\lambda_{\text {ph }}=(h c) /\left(k_{B} T\right)$ exceeds the transverse dimensions at the lowest temperatures. The speed of sound in the thin aluminum beams is $c=\sqrt{E / \rho} \approx 5060 \mathrm{~m} / \mathrm{s}$, where the Young's modulus $E=69 \mathrm{GPa}$ and density $\rho=2.7 \mathrm{~g} / \mathrm{cm}^{3}$ of aluminum have been used. This gives $\lambda_{\text {ph }} \approx 240 \mathrm{~nm}$ at $1 \mathrm{~K}$, so the thermal phonon wavelength exceeds our devices' thickness at temperatures below $1 \mathrm{~K}$. As the temperature is lowered further, the narrow devices (Fig. 1, left) cross over to the $1 \mathrm{D}$ regime, while the wide devices (Fig. 1, right) remain in the $2 \mathrm{D}$ regime down to the lowest temperatures studied in this paper. As the Fermi wavelength is small $(<1 \mathrm{~nm})$, the electrons remain $3 \mathrm{D}$ at all temperatures.

\section{TTLS damping}

The relevant damping mechanism due to TTLSs in mechanical resonators is the relaxation absorption [18,40,41]. Relaxation absorption occurs when the energy levels of the TTLS are modulated by the time-varying elastic strain field The change in the energy levels results in an instantaneous population inequilibrium which strives to relax to the equilibrium value with the relaxation time $T_{1}$. The relaxation occurs via interaction with other degrees of freedom of the system 
and causes an irreversible flow of energy, which is seen as extra damping on the mechanical devices.

At the lowest temperatures, the relaxation time is long compared to the oscillation period of the strain field. Consequently, the damping is small. As the temperature increases, the relaxation time becomes shorter and the damping increases. In this temperature range, TTLSs lead to the damping $\Gamma \propto T^{\alpha}$, where the exponent $\alpha$ depends on whether TTLSs couple dominantly to electrons, or phonons [40], or other TTLSs [42], and the possible energy dependence of the TTLS DOS [18]. In the case of TTLS-phonon coupling, the value of $\alpha$ depends on the functional form of the phonon DOS [18], which is different for the compressional, torsional, and flexural modes in $1 \mathrm{D}, 2 \mathrm{D}$, and $3 \mathrm{D}$ and, in general, may also depend on the stress in the structure [35]. This wide range of conditions leads to the qualitatively different behavior of damping in mechanical resonators hosting TTLSs observed in different works [19-32,42-44]. Our work clearly demonstrates the transition from 1D to 2D behavior with increasing device size in devices where TTLS damping is dominated by coupling to flexural phonon modes.

In the following, we outline the derivation for the damping rate due to the relaxation absorption mechanism via phonons in restricted dimensions. In general, the spatial distribution and orientation of the individual defects affect the exact contribution to the relaxation and frequency shift, e.g., due to the orientation dependence of the coupling strength [45]. We, however, assume a large ensemble of TTLSs, and it is sufficient to consider the spatially and orientationally averaged values. We make the approximation $(\gamma / c)^{2} \approx\left(\gamma_{l} / c_{l}\right)^{2} \approx$ $\left(\gamma_{t} / c_{t}\right)^{2}$, where $c, c_{l}$, and $c_{t}$ are the beam, longitudinal, and transverse speeds of sound, and $\gamma, \gamma_{l}$, and $\gamma_{t}$ are the respective TTLS-phonon coupling constants. In this approximation, the TTLS relaxation rate is [18]

$$
\left\langle T_{1}^{-1}(\epsilon)\right\rangle_{V} \approx \frac{1}{V} g(\epsilon / \hbar) \frac{\Delta_{0}^{2}}{\epsilon} \frac{\pi \gamma^{2}}{E \hbar^{2}} \operatorname{coth}\left(\frac{\epsilon}{2 k_{B} T}\right) .
$$

Here $V$ is the volume of the system, $g(\omega)$ is the phonon DOS, taken at the TTLS energy $\epsilon=\sqrt{\Delta^{2}+\Delta_{0}^{2}}, \Delta$ is the doublewell asymmetry, and $\Delta_{0}$ is the tunneling strength. Assuming a constant TTLS density of states $P_{0}$, the damping rate is [18]

$$
\begin{aligned}
\Gamma_{\text {rel }}(\omega)= & \frac{\omega P_{0} \gamma^{2}}{E k_{B} T} \int d \Delta d \Delta_{0} \frac{\Delta^{2}}{\Delta_{0} \epsilon^{2}} \\
& \times \operatorname{sech}^{2}\left(\frac{\epsilon}{2 k_{B} T}\right)\left\langle\frac{\omega T_{1}(\epsilon)}{1+\omega^{2} T_{1}(\epsilon)^{2}}\right\rangle_{V} .
\end{aligned}
$$

The double integral over $\Delta$ and $\Delta_{0}$ can be converted to an integral over $\epsilon$ using polar coordinates, $\Delta=\epsilon \cos (\theta), \Delta_{0}=$ $\epsilon \sin (\theta)$. At low temperatures $\omega_{0} T_{1} \gg 1$, the integral over the angle $\theta$ can be performed directly, resulting in

$$
\Gamma_{\text {rel }}\left(\omega_{0}\right) \approx \frac{2 \pi P_{0} \gamma^{4}}{3 \hbar^{2} E^{2} k_{B} T V} \int_{0}^{\infty} d \epsilon\left[\epsilon g\left(\frac{\epsilon}{\hbar}\right) \operatorname{csch}\left(\frac{\epsilon}{k_{B} T}\right)\right],
$$

where $2 \operatorname{csch}(x)=\operatorname{sech}^{2}(x / 2) \operatorname{coth}(x / 2)$, and the phonon DOS $g(\omega)$ is given below.

For the devices used in this paper, only the fundamental compressional, torsional, and flexural phonon modes need to be considered, as the hyperbolic cosecant term in Eq. (13) effectively cuts off the higher-order modes at low temperatures. The phonon DOS in 1D and 2D for the fundamental modes can be written as [18]

$$
\begin{gathered}
g_{1 \mathrm{D}}(\omega)=\frac{H}{\pi} \frac{1}{\left|v_{g}(\omega)\right|}, \\
g_{2 \mathrm{D}}(\omega)=\frac{H w}{2 \pi} \frac{\omega}{v_{p}(\omega)\left|v_{g}(\omega)\right|},
\end{gathered}
$$

where $H$ and $w$ are the length and width of the leg (see Fig. 2), and $v_{p}$ and $v_{g}$ are the phase and group velocities of the phonons. The dispersion relation for the flexural mode in a rectangular beam is

$$
\omega=k^{2} \sqrt{\frac{E I_{x}}{\rho w d}},
$$

where $I_{x}=w d^{3} / 12$ is the second moment of inertia. The corresponding group and phase velocities are

$$
v_{g}(\omega)=\sqrt{\frac{2 c \omega d}{\sqrt{3}}} \quad \text { and } \quad v_{p}(\omega)=\frac{1}{2} v_{g}(\omega) .
$$

Note that in highly stressed beams, modifications to the phonon group and phase velocities via the acoustoelastic effect [35] may need to be taken into account.

In thin beams and at low frequencies, the group and phase velocities associated with the flexural modes are much smaller than those associated with the compressive or torsional modes, and the phonon DOS is dominated by the flexural modes. Inserting the 1D DOS to Eq. (13), we obtain the damping rate for a rectangular beam in the $1 \mathrm{D}$ case:

$$
\Gamma_{\text {rel }, 1 \mathrm{D}} \approx 1.9 \frac{P_{0} \gamma^{4}}{E^{2}} \frac{1}{w d^{3 / 2}} \frac{1}{c^{1 / 2}} \frac{\left(k_{B} T\right)^{1 / 2}}{\hbar^{3 / 2}} .
$$

Similarly in 2D:

$$
\Gamma_{\text {rel }, 2 \mathrm{D}} \approx \frac{\pi^{2}}{4 \sqrt{3}} \frac{P_{0} \gamma^{4}}{E^{2}} \frac{1}{d^{2}} \frac{1}{c} \frac{k_{B} T}{\hbar^{2}} .
$$

Equations (18) and (19) apply to rectangular cantilever beams and doubly clamped beams that have the quadratic dispersion relation given in Eq. (16). As such, they apply to the feet of our $\Pi$-shaped devices. The almost rigid paddle of our device is not expected to contribute much to the damping, but it increases the effective mass and the energy stored in the oscillations of the resonator. Equations (18) and (19) are thus expected to overestimate the damping rate in our devices.

Linear temperature dependence is also expected, if TTLSs couple to electrons [40],

$$
\Gamma_{\mathrm{el}}=\frac{\pi^{3}}{24} \frac{P_{0} \gamma^{2}}{E}\left(N_{F} K V_{e}\right)^{2} \frac{k_{B} T}{\hbar},
$$

where $N_{F}$ is the electron DOS at the Fermi energy (accounting for both spin orientations), $K$ is a coupling constant describing the interaction between TTLSs and electrons and $V_{e} \sim 1 \mathrm{~nm}^{3}$ is the interaction volume of electrons. Inserting values $d=$ $100 \mathrm{~nm}$ and $K=0.1 \mathrm{eV}$ [30], we get $\Gamma_{\mathrm{el}} / \Gamma_{\text {rel,2D }} \sim 10^{5}$. Thus, the electronic contribution should dominate if TTLSs couple to electrons. 
The relaxation rate and damping increase with increasing temperature, until at high enough temperatures with $\omega_{0} T_{1} \ll 1$ the population equilibrium is established practically instantaneously. At these temperatures, and up to about $5 \mathrm{~K}$ where the tunneling state description is still valid, the damping rate saturates to the temperature-independent value

$$
\Gamma_{\text {rel }}=\frac{\pi \omega_{0}}{2} \frac{P_{0} \gamma^{2}}{E}=\frac{\pi \omega_{0}}{2} C,
$$

where $C=P_{0} \gamma^{2} / E$. The same result applies for metallic and insulating glasses [40] and does not depend on the dimensionality of the system [18].

\section{Frequency shift}

Another damping mechanism, important, e.g., in sound attenuation measurements, is resonant absorption, where the TTLS with excitation energy $\epsilon \sim \hbar \omega_{0}$ absorbs energy from the vibrational mode at the frequency $\omega_{0}$. In a driven mechanical resonator, the energy per unit frequency range associated with the mechanical mode typically exceeds that of the thermal phonons. In the strong strain field regime, the contribution from the resonant mechanism to the damping rate can be expressed as [40]

$$
\Gamma_{\text {res }}(\omega)=\pi \omega \frac{P_{0} \gamma^{2}}{E} \frac{\tanh \left(\hbar \omega / 2 k_{B} T\right)}{\left(1+I / I_{c}\right)^{1 / 2}},
$$

where $I=2 \rho e_{0}^{2} c^{3}$ is the acoustic intensity of the strain field $e_{0}$ and $I_{c}=\hbar^{2} \rho c^{3} /\left(2 \gamma T_{1} T_{2}\right)$ is the critical intensity where the acoustic-phonon energy per unit bandwidth is equal to that of the thermal phonons. Here $T_{2}$ is the dephasing time of the TTLS. At temperatures around $1 \mathrm{~K}$, typical values of $T_{1}$ and $T_{2}$ are of the order of $1 \mathrm{~ns}$ for insulating glasses, and the values increase with decreasing temperature. Even for small strain fields $e_{0} \sim 10^{-4}$, corresponding to $1 \mathrm{~nm}$ oscillation amplitude of a device of length $10 \mu \mathrm{m}$, the intensity ratio is $I / I_{c} \sim 10^{5}$, and the resonant absorption is completely saturated.

Associated with the resonant absorption mechanism is a change in the sound velocity which is obtained from the Kramers-Kronig relation [40],

$$
\Delta c(\omega)=\frac{c}{\pi} \int_{0}^{\infty} \frac{\Gamma\left(\omega^{\prime}\right)}{\omega^{2}-\omega^{\prime 2}} d \omega^{\prime},
$$

where the principal value of the integral is taken. The major contribution to the integral comes from the states $\hbar \omega^{\prime} \sim k_{B} T$, which are not easily saturated by the mechanical motion of the device when $\hbar \omega_{0} \ll k_{B} T$ and $\Gamma\left(\omega^{\prime}\right)=\Gamma_{\text {res }}\left(\omega^{\prime}\right)$. The sound velocity shift leads to a shift in the resonance frequency of the device,

$$
\delta \omega_{0, \mathrm{res}}=\omega_{0}-\omega_{0, r}=\omega_{0} C \ln \left(\frac{T}{T_{r}}\right),
$$

where $\omega_{0, r}$ is the resonance frequency taken at the reference temperature $T_{r}$.

Another contribution to $\Gamma$ in Eq. (23) is relaxation absorption. Relaxation absorption in 1D insulating glasses is considered in Ref. [41]. Evaluated for our devices, the model in Ref. [41] predicts a decrease in frequency $\delta \omega_{0, \text { rel, } 1 \mathrm{D}} \propto$ $T^{-1 / 6}$ at temperatures above $10 \mathrm{mK}$, but the expected frequency shift is small compared to the contribution from the resonant mechanism. To our knowledge, prediction for the frequency shift in the $2 \mathrm{D}$ case is not found in the literature. In the models for 3D phonons and electrons, the frequency shift due to the relaxation mechanism in the low-temperature regime is small compared to the resonant mechanism, and at high temperatures where $\omega_{0} T_{1} \ll 1$, the shift from the relaxation mechanism is [40]

$$
\delta \omega_{0, \mathrm{rel}, 3 \mathrm{D}}=-\frac{3}{2} \omega_{0} C \ln \left(\frac{T}{T_{r}}\right)
$$

for the phonons, and

$$
\delta \omega_{0, \mathrm{rel}, \mathrm{el}}=-\frac{1}{2} \omega_{0} C \ln \left(\frac{T}{T_{r}}\right)
$$

for the electrons. Note that $C$ is the same in Eqs. (21) and (24)(26). However, the product $P_{0} \gamma^{2}$ inferred from the measured frequency shift is often different from the one obtained from the damping, as different energy ranges are important for the different processes, and the product $P_{0} \gamma^{2}$ depends weakly on the energy [40].

The total frequency shift is the sum of the resonant and relaxation contributions $\delta \omega_{0}=\delta \omega_{0 \text {,res }}+\delta \omega_{0 \text {,rel }}$. In the low-temperature regime, the contribution from the relaxation mechanism is small and the frequency increases due to the resonant term. In the high-temperature regime, the relaxation mechanism produces a negative frequency shift. For 3D insulating glasses, the relaxation mechanism starts to dominate over the resonant mechanism around temperature $T^{*} \propto \omega_{0}^{1 / 3}$, where $\omega_{0} T_{1} \sim 1$ [40], and a maximum in the frequency is observed. The maximum in frequency in $3 \mathrm{D}$ insulating glasses occurs approximately at the same regime where the damping starts to saturate. For electrons and 1D phonons, the contribution to the frequency shift from the relaxation mechanism is smaller than the resonant contribution in both temperature regimes. In typical metallic glasses, a maximum in frequency occurs around $2-3 \mathrm{~K}$ when phonons start to dominate the relaxation process [40]. In the NEMS devices, the maximum occurs as either the $2 \mathrm{D}$ phonon process or the $3 \mathrm{D}$ phonon process starts dominating the relaxation process.

\section{RESULTS}

In the Theory section, the convenient unit of frequency is radian $(\omega, \Gamma)$, but in the experimental section it is Hertz $(f$, $\Delta f)$. Thus, in the following we use the conventions $\omega_{i}=2 \pi f_{i}$ and $\Gamma_{i}=2 \pi \Delta f_{i}$, where $i$ is the appropriate subscript $(0, r$, rel, etc.) for the given quantity.

The response of the NEMS devices has been measured in vacuum at temperatures from $16 \mathrm{mK}$ to $4 \mathrm{~K}$. The resonance properties are obtained by fitting Eqs. (5) and (6) to the measured responses, as shown in Fig. 3. The wide devices (W1, W2) are always operated well in the linear regime, allowing us to use the simple Lorentzian expressions $(\beta=0)$ in the fitting. The response of the narrow devices $(\mathrm{N} 1, \mathrm{~N} 2)$ becomes slightly nonlinear at the lowest temperatures, and we fit the response to the full equations with finite $\beta$. The absence of heating effects due to the excitation current is verified from the independence of the damping and the linear resonance frequency 

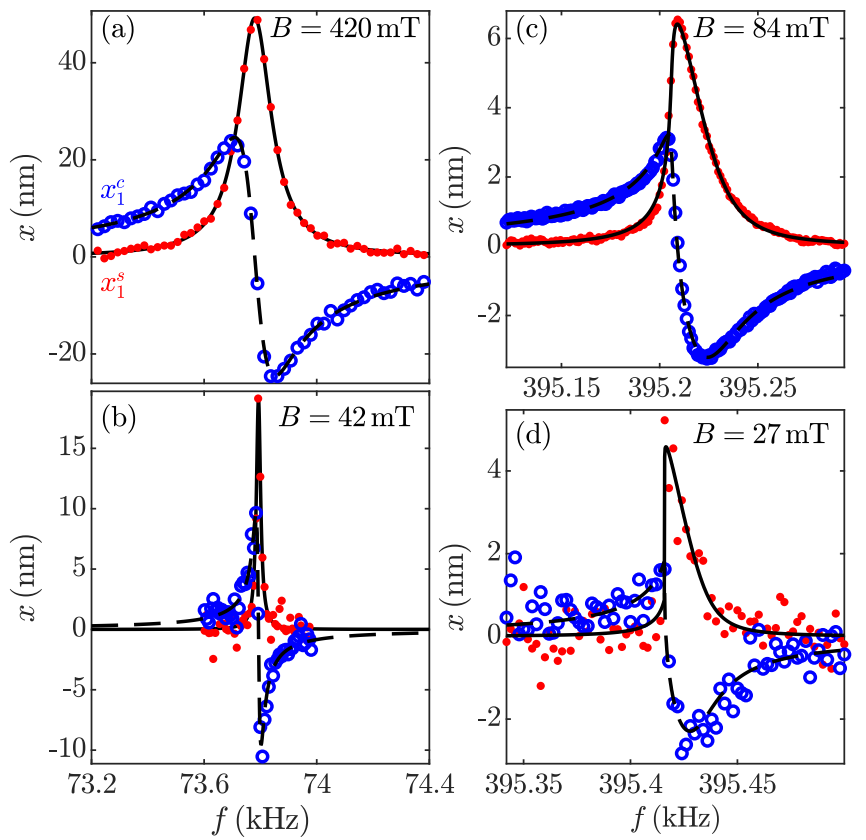

FIG. 3. Examples of the measured responses of the NEMS resonators. The damping rate $\Delta f$ and the resonance frequency $f_{0}$ of the devices are extracted by fitting Eqs. (5) and (6) to the data. (a), (b) Response of device $\mathrm{W} 1$, measured at $23 \mathrm{mK}$ at two different magnetic fields $B$ with the aluminum in the normal state. The resonance linewidth increases with increasing magnetic field due to the magnetomotive damping. Note the common frequency range. (c), (d) Response of device $\mathrm{N} 1$ measured at $16 \mathrm{mK}$ at two magnetic fields. Note the different frequency ranges. At $B=84 \mathrm{mT}$, the aluminum is in the normal state. The response is slightly asymmetric due to the nonlinearity, but the oscillation amplitude is still below the limit where bistability occurs. At $B=27 \mathrm{mT}$, the aluminum is in the superconducting state and the resonance frequency is higher than in the normal state.

on the excitation current at the lowest temperatures. This also confirms that the observed nonlinearity $\beta \sim 0.1 \mathrm{~Hz} / \mathrm{nm}^{2}$ does not affect the damping.

\section{A. Magnetic-field dependence}

The response of the devices has been measured as a function of the magnetic field. We find qualitative agreement with the $B^{2}$ dependence expected from Eqs. (8) and (9). However, using typical values $\operatorname{Re}\left(Z_{\text {ext }}\right) \sim 1 \mathrm{k} \Omega$ and $\operatorname{Im}\left(Z_{\text {ext }}\right) \sim-100 \Omega$, we find that the predicted effect for the damping is several orders of magnitude lower than what is observed in the experiments. Also, the magnetomotive damping was found to be independent of the external circuit impedance, which was adjusted by changing the resistor in the excitation line (the resistance was varied between $0.4 \mathrm{k} \Omega$ and $10 \mathrm{k} \Omega$ ). These results indicate that the ohmic losses occur within the devices rather than in the external circuit.

The magnetomotive damping is characterized by fitting the empirical model Eq. (10) to the data, as shown in Fig. 4. The measurements at a few different temperatures confirm that the magnetomotive damping effect is independent of the temperature within the measurement accuracy. However,

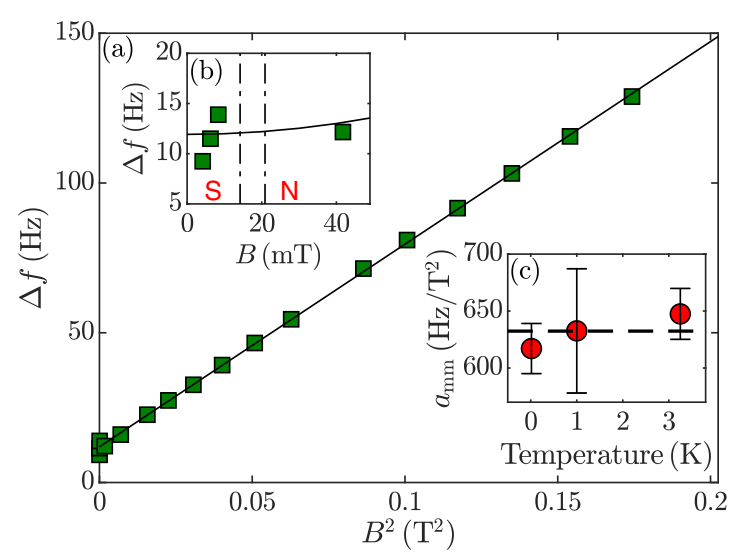

FIG. 4. (a) Damping (squares) as a function of the magnetic field for device $\mathrm{W} 1$ at $16 \mathrm{mK}$. The normal-state damping is fit to Eq. (10) (solid line), and the intrinsic damping is given by the zero-field intercept of the model. (b) Close-up of the data and fit at low magnetic fields. The vertical lines mark the transitions between the fully superconducting (S) and fully normal (N) states. The damping, measured in the superconducting state at fields $4.2 \mathrm{mT}, 6.3 \mathrm{mT}$, and $8.4 \mathrm{mT}$ are close to the zero-field intercept. (c) The fits to the measured damping at different temperatures show that the magnetomotive damping is independent of the temperature within the measurement accuracy.

changes in the parameter $a_{m}$ up to $10 \%$ have been observed as a result of thermal cycling between room temperature and base temperature. The parameter $a_{m}$ obtained from the fits is tabulated in Table I. The intrinsic damping at any temperature is determined by subtracting the fitted magnetomotive contribution from the measured damping rate.

In addition to the temperature-independent magnetomotive damping, the wide devices demonstrated another temperatureindependent contribution to the damping $\Delta f_{c}$ (tabulated in Table I), observed as a finite value of the damping when the normal-state data was extrapolated to zero temperature and zero magnetic field. This temperature-independent contribution could result, for instance, from support losses [46] or electron contributions [47] to the damping.

To determine whether electron-assisted damping is important in our devices, measurements in the superconducting state were conducted. The transition from the normal to the superconducting state is observed as a change in the electrical background signal as the resistance of the device goes to zero. For the narrow devices, the transition occurs between 28 and $32 \mathrm{mT}$ and for the wide devices between 14 and $21 \mathrm{mT}$, where the difference is due to the different thicknesses of the films [48]. The transition has a finite width in magnetic field probably due to inhomogeneity in the film. The resonance frequency is higher in the superconducting state, as the devices expel the external magnetic field, which increases the effective spring constant, see Fig. 3. The normal-superconducting transition does not have a significant effect on the damping, as seen in Fig. 4, indicating that electrons do not play a crucial role in the damping in our devices. The absence of electron-assisted damping is in line with experiments in other freestanding polycrystalline aluminum resonators, Ref. [43], but is in contrast to Refs. [24,30,47], where aluminum is deposited on silicon or silicon nitride. 

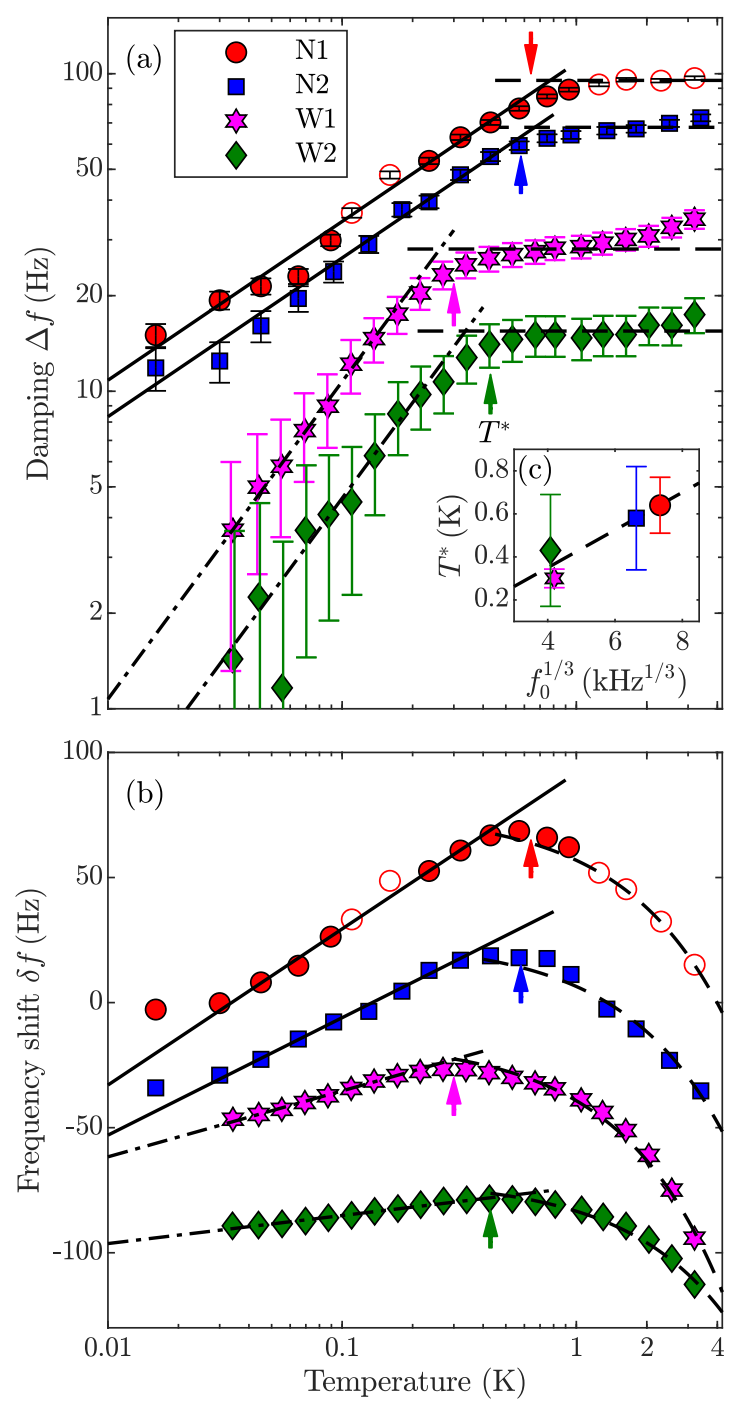

FIG. 5. (a) Damping as a function of temperature, measured for the four different devices. The error bars include uncertainty in the temperature-independent contributions that have been subtracted from the data (see main text). The solid lines are fits to the 1D model, Eq. (18), and the dash-dot lines are fits to the 2D model, Eq. (19), valid at low temperatures. The dashed lines are fits to Eq. (21) in the high-temperature regime. (b) Frequency shift as a function of temperature. Error bars are smaller than the symbol size. Data sets are shifted vertically so overlap is avoided. The empty symbols for device N1 indicate data collected after the frequency jump (see main text for details). The solid and dash-dot lines are fits to Eq. (24) at low temperatures and the dashed lines are linear fits to the hightemperature data. The arrows indicate the position of the maximum in the frequency, $T^{*}$, which marks the crossover between the lowtemperature and high-temperature regimes. The same temperatures are also marked in panel (a). (c) $T^{*}$ as a function of the cube root of the resonance frequency. The dashed line is the model $T^{*} \propto f_{0}^{1 / 3}$ fit to the data.

\section{B. TTLS damping}

The temperature-dependent damping and frequency shift of the NEMS devices, measured in vacuum, is shown in Fig. 5. The temperature-independent contributions to the damping, $a_{m} B^{2}$ and $\Delta f_{c}$, have been subtracted from the data. The relatively large error bars in the damping data reflect the uncertainty in evaluating the temperature-independent contributions.

The data for devices N1 and N2 were collected over several days, as we also measured the excitation dependencies at different temperatures. At some moment, the device N1 frequency increased in a step by approximately $15 \mathrm{~Hz}$, perhaps due to a particle that detached from the device when driving the device at a higher amplitude. However, there is no evident effect on the damping from the incident. The data collected after the frequency jump are marked with empty symbols in Fig. 5, and the corresponding frequency values are shifted down by $15 \mathrm{~Hz}$ to match earlier measured data.

At low temperatures $\left(T \lesssim T^{*}\right)$, the measured damping rate follows the model for TTLS coupling to flexural phonons in restricted dimensions. The narrow devices $\mathrm{N} 1$ and $\mathrm{N} 2$ are quasi-1D, exhibiting the $T^{1 / 2}$ temperature scaling of the damping [Eq. (18)] whereas the wide devices W1 and W2 are quasi-2D with a linear temperature dependence Eq. (19). At higher temperatures, the damping rate saturates to the temperature-independent value as expected from Eq. (21). Simultaneous fitting of Eqs. (18) or (19) to the low-temperature data and Eq. (21) to the high-temperature data gives independent values for the TTLS DOS $P_{0}$ and the TTLS-phonon coupling parameter $\gamma$. The fitted values for the different devices are tabulated in Table I. They agree with expectations based on existing literature.

The dependence of the measured damping on the effective dimensionality of the device phonon modes originates in the relaxation TTLS damping mechanism at low temperatures. At high temperatures, damping becomes insensitive to dimensionality of the device, Eq. (21), and the resonant TTLS contribution to the frequency change at low temperatures, Eq. (24), is independent of dimensionality as well. Thus we can use the product $P_{0} \gamma^{2}$ obtained from the fits of Eqs. (21) and (24) to the respective regimes as an indicator of the quality of the material in different devices. The value of $P_{0} \gamma^{2}$ turns out to be similar in wide and narrow devices, showing that the quality of the aluminum in the devices is unaffected by the subtle differences in the fabrication process.

In general, the particular values of $P_{0}$ and $\gamma$ obtained from fits may be affected by the simplifications used in our models. For example, the dispersion relation for the flexural modes, Eq. (16), is not strictly valid for goal-post-shaped devices. Also, the TTLSs are not uniformly distributed, but they are concentrated in the amorphous oxide layer and possibly at the aluminum grain boundaries. In addition, the TTLS-phonon coupling constant $\gamma$ could be different for the flexural 1D and 2D phonon modes and the bulk phonon modes. Even with these limitations, the proposed models nevertheless describe the behavior of our devices well.

In principle, the observed linear temperature dependence of the damping for the wide devices W1 and W2 might result from TTLS coupling to electrons, Eq. (20), as the measurements are conducted with the aluminum in the normal state. However, we see only a minor decrease in the damping upon transitioning to the superconducting state, even at the lowest temperature, Fig. 4, indicating that the phonon process is the dominant mechanism in our devices. The observation is in contrast to the experimental results with aluminum-covered NEMSs in Refs. [24,30], where a clear change in the damping 
between the superconducting and normal states was observed. When Eq. (20) is fitted to the damping of devices W1 and W2, the TTLS-electron coupling constant turns out to be very small, $K \sim 10^{-4} \mathrm{eV}$, compared to the value $K \sim 0.1 \mathrm{eV}$ claimed in Ref. [30]. The difference is that our resonators are made of bare aluminum, while the device in Ref. [24] is aluminum-covered silicon and the device in Ref. [30] is aluminum-covered silicon nitride. This result indicates that Al-Si and Al-SiN interfaces [24,30] act like amorphous metals where TTLSs couple to electrons, whereas in the bare aluminum devices the TTLSs mostly reside within or at the surface of the oxide layer which acts as an amorphous insulator. The number of TTLSs at the grain boundaries of the aluminum metal must be very small in our devices or their coupling to electrons must be very weak.

At $T \lesssim T^{*}$, the frequency shift is fit to the logarithmic model, Eq. (24), and the fits are shown as lines in Fig. 5. The product $P_{0} \gamma^{2}$ obtained from the measurements of the damping is about twice higher than that obtained from the frequency-shift measurement at low temperatures (see Table I). The difference probably originates from the different energy regimes probed by the two relevant processes [40]. The crossover temperature $T^{*}$ is taken as the position of the maximum of the resonance frequency, as shown in Fig. 5. The $T^{*}$ obtained from the frequency-shift data match well with the onset of the saturated-damping regime. The crossover temperature scales with the resonance frequency as $T^{*} \propto f_{0}^{1 / 3}$ (dashed line in Fig. 5 inset), which agrees with phononmediated TTLS relaxation in bulk insulating glasses [40]. The relevance of the bulk scaling to our results indicates that at $T \gtrsim T^{*}$ the phonon wave length becomes short, and the devices leave the regime of restricted dimensionality. At sufficiently high temperatures, the frequency shift should be given by the 3D model, Eq. (25). However, above $1 \mathrm{~K}$, contributions from higher-order phonon modes result in excess relaxation, which leads to the observed approximately linear decrease of the frequency with temperature [40].

\section{CONCLUSIONS}

We have studied the intrinsic damping mechanisms in magnetomotively driven aluminum nanomechanical resonators of various sizes at millikelvin temperatures. The resonators can be used as ultrasensitive sensors in a wide range of applications, e.g., in studying superfluids $[13,49]$ including quantized vortices [12], and the correct interpretation of the results requires a good understanding of the underlying device properties. The most significant mechanisms are found to be the damping due to the TTLSs and the magnetomotive damping.

As the dimensions of the nanomechanical resonators are small compared to the relevant phonon wavelengths at low temperatures, the bulk model of the TSM is not valid, and modifications to the allowed phonon modes need to be accounted for. We find good agreement with a model where TTLSs couple to flexural phonons that are geometrically restricted to one or two dimensions depending on the size of the device [18]. The model can be used as an aid when optimizing the geometrical parameters of nanomechanical resonators for sensor applications, and can be applied to other systems exhibiting TTLSs.
We find that the damping is similar in the superconducting and the normal state, indicating that electrons do not contribute significantly to the damping in the suspended aluminum, in contrast to other results reported in the literature $[24,30]$, where aluminum is deposited on silicon or silicon nitride. In our devices, the upper limit to electron-TTLS coupling is below $10^{-4} \mathrm{eV}$, which is at least three orders of magnitude smaller than in devices with deposited aluminum. The result suggests that the performance of qubits based on superconducting aluminum can possibly be improved by suspending them. This goes along with the demonstration in Ref. [50], where longer decoherence times for suspended qubits were observed.

For the magnetomotive damping mechanism, we find that in the goal-post geometry with relatively wide paddle, dissipative currents within the moving beam are important, whereas dissipation in the external circuit is negligible. The magnetomotive damping can be reduced by operating the device in a low magnetic field or with the aluminum in the superconducting state at the cost of the signal-to-noise ratio. The highest force sensitivity is obtained at the optimal magnetic field, where the contribution from the magnetomotive damping effect is equal in magnitude to the other damping mechanisms. At the lowest temperatures, the resonance line width becomes narrow, and nonlinear effects are already seen at rather low oscillation amplitudes. The magnetomotive damping effect allows one to extend the linear operating regime of the devices simply by increasing the magnetic field.

Beyond nanomechanical resonators, noise and dissipation from TTLSs affect a wide range of quantum-limited measurements, e.g., in qubits and in optomechanical systems. Our findings may aid in their analysis and design.

\section{ACKNOWLEDGMENTS}

We thank Daniel Cox and Laure Mercier de Lepinay for the support in the device fabrication, Igor Todoschenko and Jukka-Pekka Kaikkonen for the support in setting up the low-temperature experiments, and Eddy Collin for the fruitful discussions. We acknowledge the technical support from Micronova Nanofabrication Centre of VTT. This work has been supported by the European Research Council (ERC) under the European Union's Horizon 2020 research and innovation programme (Grant Agreement No. 694248) and by Academy of Finland (Grant No. 332964). The research leading to these results has received funding from the European Union's Horizon 2020 research and innovation programme under Grant Agreement No. 824109. The experiments were performed at the Low Temperature Laboratory, which is a part of the OtaNano research infrastructure of Aalto University and of the EU H2020 European Microkelvin Platform. T.K. acknowledges financial support from the Finnish Cultural Foundation (Grants No. 00190453, No. 00201211, and No. 00212577).

\section{APPENDIX: ESTIMATION OF EDDY CURRENT DAMPING DUE TO A VELOCITY GRADIENT}

Considering the geometry of our devices (see Fig. 2) and assuming the homogeneous magnetic field $\mathbf{B}=B \hat{y}$, the gen- 
erated potential at any point within the paddle is given by

$$
U(y, z)=\dot{x}(y) B z,
$$

where $H \leqslant y \leqslant(H+w), 0 \leqslant z \leqslant L$, and $z=0$ is chosen as the zero-potential reference. As a first approximation, the velocity profile of the paddle is given by

$$
\dot{x}(y)=\frac{\dot{x}_{\max }}{H+w} y,
$$

where the maximum velocity $\dot{x}_{\max }$ is attained at the outer edge of the paddle. As a result, an electric field in the $y$ direction forms, with the magnitude

$$
E_{y}(z)=-\frac{\mathrm{d} U}{\mathrm{~d} y}=-\frac{\dot{x}_{\max }}{H+w} B z .
$$

The electric field in the $z$ direction is balanced by the magnetic force $\dot{\mathbf{x}} \times \mathbf{B}$ on the electrons. Thus, there is no current in the $z$ direction. For electron motion along the field lines in the $y$ direction, the magnetic force is zero and the electric field leads to the current density $J_{y}=\sigma E_{y}$, where $\sigma$ is the electrical conductivity. The corresponding dissipated power is obtained from

$$
P_{m}=\int_{V} \mathbf{J} \cdot \mathbf{E} d V=\int_{V} \sigma E_{y}^{2} d V
$$

where the integration is taken over the volume of the paddle. In terms of the damping rate, the dissipated power is $P_{m}=m_{0} \Gamma_{m} \dot{x}_{\max }^{2}$. By evaluating the integral, we get

$$
\Gamma_{m}=\frac{1}{3} \frac{L^{2} B^{2}}{R_{t} m_{0}}\left(\frac{w}{H+w}\right)^{2},
$$

where $R_{t}=\rho_{e} w /(L d)$ is the transverse resistance of the paddle in the $y$ direction and $\rho_{e} \approx 2 \times 10^{-9} \Omega \mathrm{m}$ is the electrical resistivity of the aluminum in the normal state, measured at the cryostat base temperature. Comparing expressions, the result in Eq. (A5) is obtained from Eq. (8) if the effective circuit impedance $Z_{\text {eff }}$ is replaced with the effective resistance of the paddle:

$$
R_{\mathrm{eff}}=3 R_{t}\left(\frac{H+w}{w}\right)^{2} .
$$

Inserting device parameters to Eq. (A5) and approximating the transverse resistance with the sheet resistance, $R_{t} \approx R_{S}=$ $\rho_{e} / d$, we get $\Gamma_{m} /\left(2 \pi B^{2}\right) \sim 1 \mathrm{kHz} / \mathrm{T}^{2}$, which is of the same order of magnitude as the experimentally determined values $a_{m}$ tabulated in Table I.

We note that this simple approach ignores the dissipation during charge separation process, assumes currents uniform along the $y$ axis, while in reality eddies are more likely to be formed, and ignores the magnetic field generated by the eddy currents. Nevertheless, it results in the realistic estimation of damping, unlike the model of Eq. (8), which assumes damping in the external circuit.
[1] D. Rugar, R. Budakian, H. J. Mamin, and B. W. Chui, Single spin detection by magnetic resonance force microscopy, Nature (London) 430, 329 (2004).

[2] A. D. O'Connell, M. Hofheinz, M. Ansmann, R. C. Bialczak, M. Lenander, E. Lucero, M. Neeley, D. Sank, H. Wang, M. Weides, J. Wenner, J. M. Martinis, and A. N. Cleland, Quantum ground state and single-phonon control of a mechanical resonator, Nature (London) 464, 697 (2010).

[3] M. González, P. Zheng, E. Garcell, Y. Lee, and H. B. Chan, Comb-drive micro-electro-mechanical systems oscillators for low temperature experiments, Rev. Sci. Instrum. 84, 025003 (2013).

[4] M. Defoort, S. Dufresnes, S. L. Ahlstrom, D. I. Bradley, R. P. Haley, A. M. Guénault, E. A. Guise, G. R. Pickett, M. Poole, A. J. Woods, V. Tsepelin, S. N. Fisher, H. Godfrin, and E. Collin, Probing Bogoliubov quasiparticles in superfluid ${ }^{3} \mathrm{He}$ with a 'vibrating-wire like' MEMS device, J. Low Temp. Phys. 183, 284 (2016).

[5] D. I. Bradley, R. George, A. M. Guénault, R. P. Haley, S. Kafanov, M. T. Noble, Y. A. Pashkin, G. R. Pickett, M. Poole, J. R. Prance, M. Sarsby, R. Schanen, V. Tsepelin, T. Wilcox, and D. E. Zmeev, Operating nanobeams in a quantum fluid, Sci. Rep. 7, 4876 (2017).

[6] C. S. Barquist, W. G. Jiang, P. Zheng, Y. Lee, and H. B. Chan, The effect of remnant vortices in He II on multiple modes of a micro-electromechanical resonator, J. Low Temp. Phys. 196, 177 (2019).

[7] C. S. Barquist, W. G. Jiang, K. Gunther, Y. Lee, and H. B.
Chan, Damping of a micro-electromechanical resonator in the presence of quantum turbulence generated by a quartz tuning fork, J. Low Temp. Phys. 201, 4 (2020).

[8] A. M. Guénault, A. Guthrie, R. P. Haley, S. Kafanov, Y. A. Pashkin, G. R. Pickett, M. Poole, R. Schanen, V. Tsepelin, D. E. Zmeev, E. Collin, O. Maillet, and R. Gazizulin, Probing superfluid ${ }^{4} \mathrm{He}$ with high-frequency nanomechanical resonators down to millikelvin temperatures, Phys. Rev. B 100, 020506(R) (2019).

[9] A. M. Guénault, A. Guthrie, R. P. Haley, S. Kafanov, Y. A. Pashkin, G. R. Pickett, V. Tsepelin, D. E. Zmeev, E. Collin, R. Gazizulin, and O. Maillet, Detecting a phonon flux in superfluid ${ }^{4} \mathrm{He}$ by a nanomechanical resonator, Phys. Rev. B 101, 060503(R) (2020).

[10] R. Blaauwgeers, M. Blazkova, M. Človečko, V. B. Eltsov, R. de Graaf, J. Hosio, M. Krusius, D. Schmoranzer, W. Schoepe, L. Skrbek, P. Skyba, R. E. Solntsev, and D. E. Zmeev, Quartz tuning fork: Thermometer, pressure- and viscometer for helium liquids, J. Low Temp. Phys. 146, 537 (2007).

[11] E. Pentti, J. Rysti, A. Salmela, A. Sebedash, and J. Tuoriniemi, Studies on helium liquids by vibrating wires and quartz tuning forks, J. Low Temp. Phys. 165, 132 (2011).

[12] A. Guthrie, S. Kafanov, M. T. Noble, Y. A. Pashkin, G. R. Pickett, V. Tsepelin, A. A. Dorofeev, V. A. Krupenin, and D. E. Presnov, Nanoscale real-time detection of quantum vortices at millikelvin temperatures, Nat. Commun. 12, 2645 (2021).

[13] T. Kamppinen and V. B. Eltsov, Nanomechanical resonators for cryogenic research, J. Low Temp. Phys. 196, 283 (2019). 
[14] M. Imboden and P. Mohanty, Dissipation in nanoelectromechanical systems, Phys. Rep. 534, 89 (2014).

[15] P. Mohanty, D. A. Harrington, K. L. Ekinci, Y. T. Yang, M. J. Murphy, and M. L. Roukes, Intrinsic dissipation in high-frequency micromechanical resonators, Phys. Rev. B 66, 085416 (2002).

[16] W. A. Phillips, Tunneling states in amorphous solids, J. Low Temp. Phys. 7, 351 (1972).

[17] P. W. Anderson, B. I. Halperin, and C. M. Varma, Anomalous low-temperature thermal properties of glasses and spin glasses, Philos. Mag. (1798-1977) 25, 1 (1972).

[18] R. O. Behunin, F. Intravaia, and P. T. Rakich, Dimensional transformation of defect-induced noise, dissipation, and nonlinearity, Phys. Rev. B 93, 224110 (2016).

[19] G. Zolfagharkhani, A. Gaidarzhy, S.-B. Shim, R. L. Badzey, and P. Mohanty, Quantum friction in nanomechanical oscillators at millikelvin temperatures, Phys. Rev. B 72, 224101 (2005).

[20] S. B. Shim, J. S. Chun, S. W. Kang, S. W. Cho, S. W. Cho, Y. D. Park, P. Mohanty, N. Kim, and J. Kim, Micromechanical resonators fabricated from lattice-matched and etch-selective GaAs/InGaP/GaAs heterostructures, Appl. Phys. Lett. 91, 133505 (2007).

[21] J. Sulkko, M. A. Sillanpää, P. Häkkinen, L. Lechner, M. Helle, A. Fefferman, J. Parpia, and P. J. Hakonen, Strong gate coupling of high-Q nanomechanical resonators, Nano lett. 10, 4884 (2010).

[22] A. Venkatesan, K. J. Lulla, M. J. Patton, A. D. Armour, C. J. Mellor, and J. R. Owers-Bradley, Dissipation due to tunneling two-level systems in gold nanomechanical resonators, Phys. Rev. B 81, 073410 (2010).

[23] F. Hoehne, Y. A. Pashkin, O. Astafiev, L. Faoro, L. B. Ioffe, Y. Nakamura, and J. S. Tsai, Damping in high-frequency metallic nanomechanical resonators, Phys. Rev. B 81, 184112 (2010).

[24] K. J. Lulla, M. Defoort, C. Blanc, O. Bourgeois, and E. Collin, Evidence for the Role of Normal-State Electrons in Nanoelectromechanical Damping Mechanisms at Very Low Temperatures, Phys. Rev. Lett. 110, 177206 (2013).

[25] Y. Tao, J. M. Boss, B. A. Moores, and C. L. Degen, Singlecrystal diamond nanomechanical resonators with quality factors exceeding one million, Nat. Commun. 5, 3638 (2014).

[26] T. Faust, J. Rieger, M. J. Seitner, J. P. Kotthaus, and E. M. Weig, Signatures of two-level defects in the temperature-dependent damping of nanomechanical silicon nitride resonators, Phys. Rev. B 89, 100102(R) (2014).

[27] S. Rebari, S. Kumar, S. Indrajeet, A. Kumar, S. P. Pal, D. Weiss, and A. Venkatesan, Tunable low-temperature dissipation scenarios in palladium nanomechanical resonators, Phys. Rev. B 95, 214113 (2017).

[28] M. Kim, J. Kim, J. Kim, S.-B. Shim, B. Kim, and J. Suh, Surface two-level state dissipation in single-crystalline gold nanomechanical resonators, J. Korean Phys. Soc. 70, 225 (2017).

[29] B. D. Hauer, P. H. Kim, C. Doolin, F. Souris, and J. P. Davis, Two-level system damping in a quasi-one-dimensional optomechanical resonator, Phys. Rev. B 98, 214303 (2018).

[30] O. Maillet, D. Cattiaux, X. Zhou, R. R. Gazizulin, O. Bourgeois, A. D. Fefferman, and E. Collin, Nanomechanical damping via electron-assisted relaxation of two-level systems, arXiv:2009.03804.
[31] G. S. MacCabe, H. Ren, J. Luo, J. D. Cohen, H. Zhou, A. Sipahigil, M. Mirhosseini, and O. Painter, Nano-acoustic resonator with ultralong phonon lifetime, Science 370, 840 (2020).

[32] E. A. Wollack, A. Y. Cleland, P. Arrangoiz-Arriola, T. P. McKenna, R. G. Gruenke, R. N. Patel, W. Jiang, C. J. Sarabalis, and A. H. Safavi-Naeini, Loss channels affecting lithium niobate phononic crystal resonators at cryogenic temperature, Appl. Phys. Lett. 118, 123501 (2021).

[33] J.-P. DesOrmeaux and C. C. Striemer, Free-standing silicon oxide membranes and methods of making and using same, U.S. Patent No. 9,945,030 (April 2018).

[34] TEMwindows.com, a division of SiMPore Inc., product code No. SO100-A40Q33.

[35] B. Graczykowski, J. Gomis-Bresco, F. Alzina, J. S. Reparaz, A. Shchepetov, M. Prunnila, J. Ahopelto, and C. M. S. Torres, Acoustic phonon propagation in ultra-thin Si membranes under biaxial stress field, New J. Phys. 16, 073024 (2014).

[36] E. Collin, Y. M. Bunkov, and H. Godfrin, Addressing geometric nonlinearities with cantilever microelectromechanical systems: Beyond the Duffing model, Phys. Rev. B 82, 235416 (2010).

[37] A. N. Cleland and M. L. Roukes, External control of dissipation in a nanometer-scale radiofrequency mechanical resonator, Sens. Actuators, A 72, 256 (1999).

[38] D. Jiles, Introduction to Magnetism and Magnetic Materials, 3rd ed. (CRC Press, Boca Raton, 2015).

[39] M. Bonnet and E. Demaldent, The eddy current model as a lowfrequency, high-conductivity asymptotic form of the Maxwell transmission problem, Comput. Math. Appl. 77, 2145 (2019).

[40] W. A. Phillips, Two-level states in glasses, Rep. Prog. Phys. 50, 1657 (1987).

[41] C. Seoánez, F. Guinea, and A. H. Castro Neto, Surface dissipation in nanoelectromechanical systems: Unified description with the standard tunneling model and effects of metallic electrodes, Phys. Rev. B 77, 125107 (2008).

[42] A. D. Fefferman, R. O. Pohl, A. T. Zehnder, and J. M. Parpia, Acoustic Properties of Amorphous Silica Between 1 and 500 mK, Phys. Rev. Lett. 100, 195501 (2008).

[43] R. König, P. Esquinazi, and B. Neppert, Tunneling systems in polycrystalline metals: Absence of electron-assisted relaxation, Phys. Rev. B 51, 11424 (1995).

[44] J. Classen, T. Burkert, C. Enss, and S. Hunklinger, Anomalous Frequency Dependence of the Internal Friction of Vitreous Silica, Phys. Rev. Lett. 84, 2176 (2000).

[45] D. V. Anghel, T. Kühn, Y. M. Galperin, and M. Manninen, Interaction of two-level systems in amorphous materials with arbitrary phonon fields, Phys. Rev. B 75, 064202 (2007).

[46] D. M. Photiadis and J. A. Judge, Attachment losses of high Q oscillators, Appl. Phys. Lett. 85, 482 (2004).

[47] O. Suchoi and E. Buks, Damping in a superconducting mechanical resonator, Europhys. Lett. 117, 57008 (2017).

[48] R. Meservey and P. M. Tedrow, Properties of very thin aluminum films, J. Appl. Phys. 42, 51 (1971).

[49] I. Golokolenov, B. Alperin, B. Fernandez, A. D. Fefferman, and E. Collin, Fully suspended nano-beams for quantum fluids, arXiv:2111.05278.

[50] Y. Chu, C. Axline, C. Wang, T. Brecht, Y. Y. Gao, L. Frunzio, and R. J. Schoelkopf, Suspending superconducting qubits by silicon micromachining, Appl. Phys. Lett. 109, 112601 (2016). 